\title{
Acceleration of spin-orbit coupled Bose-Einstein condensates: analytical description of the emergence of Landau-Zener transitions
}

\author{
J.M. Gomez Llorente and J. Plata \\ Departamento de Física, Universidad de La Laguna, \\ La Laguna E38204, Tenerife, Spain.
}

\begin{abstract}
We analytically study the effect of gravitational and harmonic forces on ultra-cold atoms with synthetic spin-orbit coupling (SOC). In particular, we focus on the recently observed transitions between internal states induced by acceleration of the external modes. Our description corresponds to a generalized version of the Landau-Zener (LZ) model: the dimensionality is enlarged to combine the quantum treatment of the external variables with the internal-state characterization; additionally, atomic-interaction effects are considered. The emergence of the basic model is analytically traced. Namely, by using a sequence of unitary transformations and a subsequent reduction to the spin space, the SOC Hamiltonian, with the gravitational potential incorporated, is exactly converted into the primary LZ scenario. Moreover, the transitions induced by harmonic acceleration are approximately cast into the framework of the basic LZ model through a complete analytical procedure. We evaluate how the validity of our picture depends on the system preparation and on the magnitude of atomic-interaction effects. The identification of the regime of applicability and the rigorous characterization of the parameters of the effective model provide elements to control the transitions.
\end{abstract}

PACS numbers: 03.75.Lm, 67.85.De 


\section{INTRODUCTION}

The research on ultracold atoms has been significantly enriched by the realization of synthetic spin-orbit coupling (SOC), i.e., of induced interaction between the center-of-mass momentum and internal (hyperfine) states [1, 2]. The development of different variations of the basic scenario, with the application to, both, bosonic and fermionic systems [3, 4], has paved the way to an active area of research, where the advances in theory and experiments are continuous. A variety of fundamental effects have been uncovered and powerful technical implications of the findings are expected. Particularly interesting is the potential emergence in these systems of novel states of matter, like nontrivial superfluids or topological insulators [1, 5, 6]. The implementation of strategies for controlling different aspects of the dynamics is crucial for the advances in this line [7 9]. Here, we focus on recent experiments on spin-orbit coupled Bose-Einstein condensates which uncovered the possibility of using the external dynamics to manipulate the spin polarization [10]. Specifically, in a Raman-induced SOC setup [10], acceleration of the external modes due to gravitational or harmonic trapping forces was shown to induce transitions between energy bands associated with eigenvalues of the internal-state reduced Hamiltonian. In a preliminary analysis of the results [10], the detected features were found to be reproduced by the Landau-Zener (LZ) model. In particular, the measured probabilities of transition between spin states agreed with the predictions of the model in the asymptotic limit. In this sense, the study has a certain predictive power that allows the possibility of tuning the transition characteristics. Still, the explanation of some aspects of the physical mechanisms responsible for the observed behavior requires additional work. Namely, a more complete characterization of the emergence of the LZ model in those systems is needed. The role of the external dynamics in the inter-band transitions must be clarified. Also important is to explain the observed robustness of the model against variations in the characteristics of the acceleration methods. It is worth pointing out that, although the terms added to the basic SOC Hamiltonian in the two considered schemes have different functional forms, the global output is similar. An additional open question refers to the relevance of many-body effects to the transition processes. To deal with those issues, we will analytically study the two implemented setups. In our analysis, the applicability of the LZ model will be rigorously traced and the origin of the parallelism observed between the two schemes will be identified. Moreover, effects specific 
to the properties of the initial state will be tackled: we will see that, for the LZ approach to be applicable, restrictions on the system preparation must be imposed. We will also assess the robust character of the single-particle approach against atomic-interaction effects.

The outline of the paper is as follows. In Sec. II, the main characteristics of the system in the absence of acceleration schemes are summarized. Additionally, we discuss how the internal dynamics is altered when acceleration of the external modes is introduced in the basic scenario. The relevance of the LZ model to the description of different variations of the system is evaluated. In Sec. III, the effect of the gravitational field is studied. Through an appropriate sequence of unitary transformations and an ultimate reduction to the spin space, the Hamiltonian of the complete system is cast into the basic form of the LZ model. Some aspects specific to the preparation of the system and the measurement procedure are discussed. Sec. IV is dedicated to the analysis of the acceleration due to harmonic trapping forces. A method based on the eikonal approximation is applied first to put the focus on the dynamics of the transitions. Subsequently, an approximate analytical study of the evolution outside the crossing region is given to complete the picture. The relevance of many-body effects to the applicability of our approach is discussed in Sec. V. Finally, some general conclusions are summarized in Sec. VI.

\section{THE LANDAU-ZENER MODEL IN SPIN-ORBIT COUPLED ATOMS}

The inter-band transitions on which we focus can take place in different realizations

of SOC in Bose-Einstein condensates [5]. Here, without loss of generality, we will base our analysis on the setup presented in Ref. [1]. There, synthetic SOC was generated via an appropriate arrangement of Raman lasers. Specifically, one of the components of the external linear momentum of the atoms $\mathbf{p}$ was coupled to an effective "spin", i.e., to a two-level internal system formed by hyperfine states. The arrangement incorporated two orthogonally polarized Raman lasers with different propagation directions and frequencies. The setup was configured to couple two Zeeman-split internal states; furthermore, the coupling was made to be dependent on the atom external momentum. In this form, an effective SOC was created. Actually, in the considered experimental setup [10], as in previous standard realizations of synthetic SOC, the three states $\left|m_{F}\right\rangle$ of the hyperfine $F=1$ ground-state manifold of ${ }^{87} \mathrm{Rb}$ are coupled. It is the detuning due quadratic Zeeman shift of the state with $m_{F}=1$ that 
allows the reduction of the dynamics to the two levels with $m_{F}=0,-1$. A first picture of the dynamics is provided by a basic model where a single-particle description is applied and the effect of the harmonic confinement is ignored. Intense work on different implementations of SOC, which include variations in the coupling and dimensionality, is being carried out. Here, as in the experimental realization of [10], we will focus on the setup corresponding to Rashba-Dresselhaus SOC. In the rest of the paper, the term SOC will refer exclusively to that type of coupling. The associated Hamiltonian is given by

$$
H_{S O}=\frac{P_{x}^{2}}{2 m}+\frac{\hbar \Omega}{2} \sigma_{x}+\left(\frac{\hbar \delta_{0}}{2}+\frac{\alpha_{0} P_{x}}{\hbar}\right) \sigma_{z}
$$

The atomic mass is denoted by $m, \sigma_{i}(i=x, y, z)$ are the Pauli matrices corresponding to the pseudospin, i.e., to the considered effective two-level system, and $P_{x}$ stands for the momentum operator in the coupling direction. The additional parameters refer to the laser characteristics: $\Omega$, usually termed as the offset, represents the Raman coupling amplitude, $\delta_{0}$ denotes an adjustable detuning, and $\alpha_{0}$ is the strength of the realized $\operatorname{SOC}\left(\alpha_{0}=2 E_{r} / k_{r}\right.$ where $E_{r}=\hbar^{2} k_{r}^{2} / 2 m$ and $k_{r}=2 \pi \sin (\theta / 2) / \lambda$ are, respectively, the recoil energy and momentum; $\lambda$ is the reference laser wavelength, and, $\theta$ is the angle between the directions of the Raman lasers, which, here, as in the experiments in [1, 2], is fixed to $\theta=\pi / 2$ ).

Since $P_{x}$ is a constant of the motion, it is convenient to work in the basis $\left|p_{x}\right\rangle \otimes|\chi\rangle$, where $\left|p_{x}\right\rangle$ denotes a momentum state and $|\chi\rangle$ stands for a spin state. The consequent reduction of the Hamiltonian to the spin space is simply obtained by replacing the operator $P_{x}$ by $\hbar k_{x}$ in Eq. (11). ( $k_{x}$ is the quasi-momentum in the coupling direction, $\left.\mathbf{p}=\hbar \mathbf{k}\right)$. Using this approach, the system is found to present energy bands given by

$$
E_{ \pm}\left(k_{x}\right)=\frac{\hbar^{2} k_{x}^{2}}{2 m} \pm \sqrt{\left(\frac{\hbar \Omega}{2}\right)^{2}+\left(\frac{\hbar \delta_{0}}{2}+\alpha_{0} k_{x}\right)^{2}}
$$

Since the bands correspond to eigenvalues of the reduced Hamiltonian, no coupling between them exists in this scheme. They reflect the existence of locking between the momentum and the spin state: each quasi-momentum value is attached to a particular eigenvalue, and, in turn, to the corresponding combination of internal states. We will see that interband transfers of population can take place in variations of the above setup, specifically, in the arrangements implemented in Ref. [10]. It will be shown that the transitions can be described in terms of switching between adiabatic states in the LZ model [11, 12]. Actually, 
the adiabatic regime can be already identified: an external mechanism that can induce a slow variation of $k_{x}$ can be expected to lead to an adiabatic following of the internal state along the band, the static locking being trivially conserved in the resulting evolution.

Let us recall some basic characteristics of the LZ approach. In its simplest form, this model deals with a system of two coupled states (the diabatic states) with a linear variation of the energy mismatch. The associated Hamiltonian reads

$$
H_{1}=\hbar \frac{v t}{2} \sigma_{z}+\hbar \zeta \sigma_{x}
$$

where $v$ denotes the rate of the splitting variation, and $\zeta$ represents the coupling strength. An alternative description in terms of the adiabatic states, i.e., of the instantaneous eigenstates of the coupled system, is also convenient. Both pictures give the same information on the dynamics. We will switch from one description to the other as it can be demanded by the clarification of the physics underlying the studied transitions. At the initial and final times, which are formally defined as $t \rightarrow \mp \infty$, the energy levels are assumed to be far apart. Therefore, at those times, the adiabatic states can be considered to approximately match the diabatic states. Actually, $\zeta$ is assumed to be sufficiently small for the coupling to be effective only near the crossing that takes place between the diabatic levels at $t=0$. In those conditions, the probability of transition between diabatic states at the end of the linear ramp is given by

$$
P_{L Z}^{(d)}=1-e^{-2 \pi|\zeta|^{2} / v}
$$

The counterpart description in the adiabatic basis depicts an avoided crossing at $t=0$, the asymptotic probability of transition between adiabatic states being given by $P_{L Z}^{(a)}=e^{-2 \pi|\zeta|^{2} / v}$. Note that the adiabatic regime, characterized by $P_{L Z}^{(a)}=0$, is approached as the ratio $|\zeta|^{2} / v$ is increased. The LZ model also applies to variations of the basic scenario described by Eq. (3) which incorporate an independent term in the linearly-modified energy mismatch, i.e., to Hamiltonians with the form

$$
H_{2}=\hbar\left(\Lambda+\frac{v t}{2}\right) \sigma_{z}+\hbar \zeta \sigma_{x}
$$

Moreover, the initial and final times of the process do not need to correspond to $t \rightarrow \mp \infty$. The only restrictions are the existence of a crossing, i.e., the occurrence of a zero value of the energy mismatch during the ramping, and the need of having sufficiently large values of 
the magnitude of the initial and final splittings. Relevant to the cases that will be analyzed further on in this paper is to refer to an initial time $t_{i}=0$, and, consequently to an initial splitting given by the independent term $\hbar \Lambda$. It is apparent that a positive (negative) $\Lambda$ requires a negative (positive) ramp rate $v$ for the restrictions of the LZ model to be fulfilled. The probability of transition for the case of negative rate is obtained by simply changing $v$ by $|v|$ in Eq. (4). Hence, in the following, to account for both cases, we will respectively use the expressions $P_{L Z}^{(d)}=1-e^{-2 \pi|\zeta|^{2} /|v|}$ and $P_{L Z}^{(a)}=e^{-2 \pi|\zeta|^{2} /|v|}$ for the diabatic and adiabatic probabilities of transition.

Let us evaluate the applicability of the LZ approach to the SOC setup. In the system considered by now, see Eq. (11), the splitting between the spin states is fixed since $P_{x}$ is a constant of the motion ( $k_{x}$ enters the reduced description as a fixed parameter). In fact, a level crossing occurs only for a specific value of the quasi-momentum $k_{x, c}$, namely, for that given by the condition

$$
k_{x, c}=-\frac{\hbar \delta_{0}}{2 \alpha_{0}}
$$

Hence, $k_{x, c}$ is determined by the detuning and by the SOC strength; in particular, $k_{x, c}=0$, for zero detuning. Obviously, no analogy with the LZ model can be traced at this point. However, we can think of variations of the above static scenario where a modification of the spin-state splitting can be implemented via the control of the external dynamics. Specifically, one can propose setups where the acceleration of the system in the $x$-direction can be used to vary the energy mismatch. This is the case of the situations considered in the experiments reported in [10]: the acceleration mechanisms were incorporated to drive the momentum to the range of values where $k_{x} \simeq k_{x, c}$. There, the coupling incorporated in the offset becomes effective to activate the crossing, and, consequently, transitions between internal states can be induced. Here, the applicability of the LZ model can be reasonably conjectured. The dispersion curves (dressed bands) given by Eq. (2) can be thought of corresponding to the adiabatic levels of the LZ model. The existence of an avoided level crossing is apparent in them; the magnitude of the associated gap is given by the offset: as $\Omega$ increases, the adiabatic bands progressively separate. Note that, in the adiabatic picture, it is an interband transition that is brought about by the acceleration. The diabatic counterparts (bare bands) correspond to taking $\Omega=0$ in those curves: the offset disappears from the expressions for the energy levels and incorporates the coupling between (diabatic) spin states. Whereas, 
in the primary LZ setup, it is a driving term, independent of the considered dynamics, that induces the linear variation of the energy mismatch, in our system, it is the dynamics of the external variables that leads to the modification of the internal-state splitting. In the next sections, we will analytically trace the occurrence of LZ transitions generated by gravitational and harmonic-trapping forces. Dealing with the required enlarged dimensionality of the problem is one of the aims of our work. Another basic objective is to analyze the implications to the extended system of the conditions of having large initial and final splittings, required for the applicability of the basic LZ model.

\section{GRAVITATIONAL ACCELERATION}

Let us analyze first the case where the acceleration of the system is induced by the gravitational field. In the experiments [10], that situation was arranged by implementing the SOC in the vertical axis. Then, the trap was turned off, and the system, prepared as a Gaussian superposition of momentum states, was led to evolve under the effect of the gravity. Accordingly, we consider here that, at $t=0$, the gravitational field is connected, the dynamics being then governed by the Hamiltonian

$$
H_{G}=H_{S O}+H_{\text {grav }}
$$

where

$$
H_{\text {grav }}=\operatorname{mgX}
$$

Note that, in our theoretical framework, which corresponds to non-relativistic quantum mechanics, the effect of gravity can be regarded as that of an effective external electric field. As shown in [13], this approach is convenient to operatively account for nontrivial effects of gravity on a quantum context.

In the setup provided by Eqs. (7) and (8), $P_{x}$ is not a constant of the motion. Indeed, as previously stated, the variation of the momentum, i.e., the acceleration of the system, is the

objective of incorporating $H_{\text {grav }}$. Appropriate to analyze the dynamics is the application of the unitary transformation 


$$
U_{1}(t)=\exp \left[-\frac{i}{\hbar} m g X t\right]
$$

which introduces a time-dependent displacement in momentum, specifically, it implies working in a reference frame translated with acceleration $g$. The transformed Hamiltonian, given by

$$
H_{G}^{\prime}=U_{1}^{\dagger} H_{G} U_{1}-i \hbar U_{1}^{\dagger} \dot{U}_{1}
$$

is written, after straightforward algebra [14], as

$$
\begin{aligned}
H_{G}^{\prime}= & \frac{P_{x}^{2}}{2 m}-g P_{x} t+\frac{\hbar \Omega}{2} \sigma_{x}+ \\
& \frac{\hbar \delta_{0}}{2} \sigma_{z}+\frac{\alpha_{0}}{\hbar}\left(P_{x}-m g t\right) \sigma_{z},
\end{aligned}
$$

where we have shifted the energy origin by $m g^{2} t^{2} / 2$. Now, a second unitary transformation with the form

$$
U_{2}(t)=\exp \left[-\frac{i}{\hbar} \frac{P_{x}^{2}}{2 m} t+\frac{i}{\hbar} g P_{x} \frac{t^{2}}{2}\right]
$$

is applied to incorporate the spin-independent terms present in Eq. (11). $U_{2}(t)$ parallels a gauge transformation which simply introduces a phase depending on both, momentum and time [15]. We obtain for the transformed Hamiltonian

$$
\begin{aligned}
H_{G}^{\prime \prime} & =U_{2}^{\dagger} H_{G}^{\prime} U_{2}-i \hbar U_{2}^{\dagger} \dot{U}_{2} \\
& =\frac{\hbar \Omega}{2} \sigma_{x}+\left[\frac{\hbar \delta_{0}}{2}+\frac{\alpha_{0}}{\hbar}\left(P_{x}-m g t\right)\right] \sigma_{z} .
\end{aligned}
$$

Again working in the representation $\left|p_{x}\right\rangle \otimes|\chi\rangle$, we can write for the reduction of the Hamiltonian to the spin space the expression

$$
H_{G}^{\prime \prime}=\frac{\hbar \Omega}{2} \sigma_{x}+\left[\frac{\hbar \delta_{0}}{2}+\frac{\alpha_{0}}{\hbar}\left(\hbar k_{x}-m g t\right)\right] \sigma_{z},
$$

where the quasi-momentum plays the role of a parameter. The analogy with the LZ model is now apparent: the resulting effective two-level system, coupled through the offset term, displays a linear variation of the energy mismatch. For a state with quasi-momentum $k_{x}$, 
the crossing time $t_{c}$ between the diabatic states is given by $t_{c}=\frac{\hbar \delta_{0} / 2+\alpha_{0} k_{x}}{\alpha_{0} m g / \hbar}$; therefore, it depends on both, $k_{x}$ and $\delta_{0}$. Importantly, in order to apply the restriction of having initially a large splitting, required for applying the LZ model, we must take into account that, here, the initial time is $t=0$. The fulfillment of that restriction is then guaranteed by working with a sufficiently large (positive) quasi-momentum. The comparison with Eq. (3) leads to the identification of the $\mathrm{LZ}$ characteristic parameters as

$$
\begin{aligned}
& \zeta \rightarrow \frac{\Omega}{2} \\
& v \rightarrow-\frac{2 \alpha_{0} m g}{\hbar^{2}}
\end{aligned}
$$

Hence, the coupling strength is given by the offset, and the rate of splitting variation is determined by the SOC amplitude and by the atom weight. The presence of the detuning $\delta_{0}$ and the quasi-momentum $k_{x}$ in Eq. (14) does not affect the applicability of the model. Although they alter the value of the crossing time $t_{c}$, they do not affect the asymptotic transition probability, which is still determined by the characteristic LZ parameters, provided that the splittings corresponding to the initial and final times are sufficiently large. Figure 1 illustrates these results.

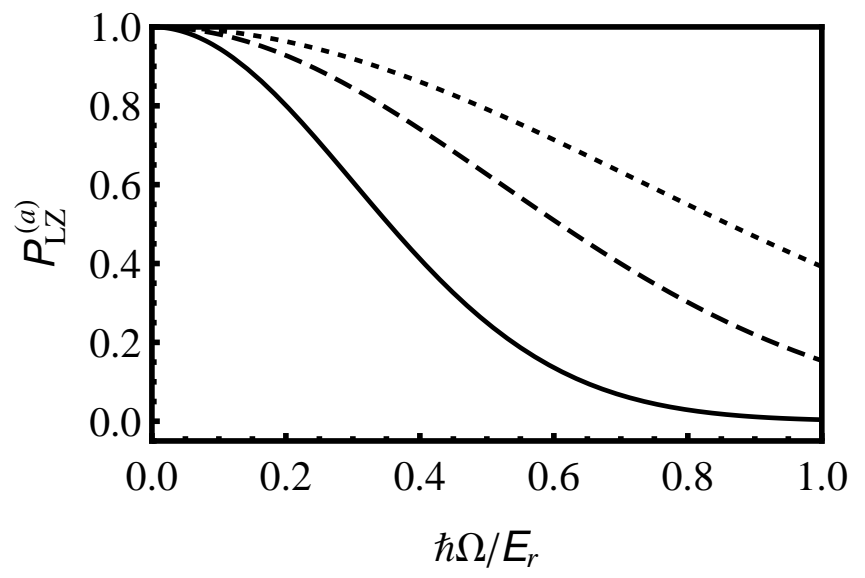

Figure 1: The probability of transition between adiabatic states as a function of the offset parameter (in units of the recoil energy, $E_{r} / 2 \pi \hbar=3.75 \mathrm{kHz}$ ). The continuous line corresponds to gravitational acceleration; The dashed line refers to harmonic acceleration with $\omega_{x} / 2 \pi=70 \mathrm{~Hz}$ and $q_{0}=1.5 \times$ $10^{-4} \mathrm{~m}$; The dotted line represents harmonic acceleration with $\omega_{x} / 2 \pi=70 \mathrm{~Hz}$ and $q_{0}=3 . \times 10^{-4}$ m. 
The above results can be applied to explain the experimental findings. The practical realization corresponded to a Gaussian superposition of momentum states. Moreover, it was the probability of transition between spin states, independently on the momentum values, that was measured. Hence, in a description in terms of the density operator of the complete system, (including, both, internal and motional variables), the experimental procedure formally corresponds to carrying out a partial trace over the momentum states. Actually, given the characteristics of the measurement procedure, it is not a pure state but a statistical mixture that is required to simulate the experimental realization. Therefore, the distribution of momentum populations of the initial preparation contains all the information necessary to reproduce the measured transition probabilities. The distribution corresponding to the experiments was assumed to be well simulated by the function

$$
\rho\left(k_{x}\right)=\frac{1}{\sqrt{2 \pi} \sigma_{w}} \exp \left[-\left(k_{x}-k_{x, i}\right)^{2} /\left(2 \sigma_{w}^{2}\right)\right],
$$

where the center $k_{x, i}$ and the width $\sigma_{w}$ of the Gaussian are parameters specific to each realization. The total probability of transition is obtained by averaging, over the distribution, the partial probabilities for the different momenta, all of them given by the LZ formula $P_{L Z}^{(d)}=1-e^{-2 \pi|\zeta|^{2} /|v|}$. In the assumed conditions, although there is a variety of crossing times, there is a unique value of the asymptotic transition probability. We recall that no amplitudes of transition are evaluated. The parameters $k_{x, i}$ and $\sigma_{w}$ must be chosen in order to guarantee that the initial splittings, for all the states that contribute to the wave-packet, are sufficiently large. Moreover, the measurement must be carried out when the momenta have reached large-magnitude values. Consequently, the system can be assumed to be far from the diabatic crossing at the initial and final times, as required for applying the standard LZ approach. These restrictions are expressed as

$$
\left|\frac{\hbar \delta_{0}}{2}+\alpha_{0}\left(k_{x, i} \pm \sigma_{w}\right)\right| \gg \frac{\hbar \Omega}{2},
$$

for the parameters of the initial distribution, a similar equation being applicable to the final momentum values. The above expression has been obtained taking into account that the crossing region corresponds to an energy mismatch comparable to the magnitude of the coupling term $\frac{\hbar \Omega}{2}$. Note the importance of the magnitude of $\Omega$ in validity of the model. The relevance of the SOC parameter $\alpha_{0}$ must also be pointed out. For a direct comparison with the parameters used in the characterization of the experimental setup, we must recall the 
dependence of $\alpha_{0}$ on the recoil energy and momentum, namely, $\alpha_{0}=2 E_{r} / k_{r}$. Note that as $\alpha_{0}$ decreases, the magnitude of the SOC diminishes, and, eventually, the effect vanishes. Then, a significant value of $\alpha_{0}$ is needed for our characterization of the system to be meaningful. One should also take into account that $\delta_{0}$ affects the location of the crossing point; additionally, it enters Eq. (17), which guarantees the validity of the LZ model, i.e., the accomplishment of the asymptotic regime in both initial and final configurations. We will go back to this point in different parts of the paper given the importance of the definition of the asymptotic regimes. In all the experimental realizations of [10], those restrictions are amply fulfilled.

In passing, our analysis provides an understanding for one conspicuous feature detected in the experiments. Namely, the observed lack of dependence of the probability of transition on the detuning is explained by the mere applicability of the LZ model: the detuning and the initial values of $k_{x}$, are irrelevant to the model predictions for the asymptotic probabilities.

Two additional remarks are pertinent. First, we have assumed that the diabatic states can be exactly identified with the internal states of the SOC Hamiltonian. In fact, in the experiments, the initial state corresponded to a combination of the two internal states with one of the associated coefficients having a magnitude much larger than the other. Our approach is still applicable to that situation: the output of the process is then a combination of states where the squared moduli of the coefficients have asymptotic values given by the predictions of the LZ for the counterpart probabilities of transition. Second, the effective rate of splitting variation $v$ is fixed since it is determined by the gravitational acceleration and by the SOC strength $\alpha_{0}$. Therefore, there is no form of reaching an adiabatic evolution by reducing $v$. The only nontrivial possibility of realizing an effective adiabatic regime, i.e.,

of diminishing $P_{L Z}^{(a)}=e^{-2 \pi|\zeta|^{2} /|v|}$, is by increasing the offset. We stress that the enhancement in $\Omega$ needed to reach the adiabatic regime can be made to be compatible with the restriction given by Eq. (17). Indeed, this is the case of the experimental realization of the adiabatic regime [10].

\section{HARMONIC ACCELERATION}

Let us consider now the case where the acceleration of the system is induced by the trapping potential. Accordingly, the dynamics is assumed to be governed by the Hamiltonian 


$$
H_{T}=H_{S O}+H_{\text {trap }}
$$

where

$$
H_{\text {trap }}=\frac{1}{2} m \omega_{x}^{2} X^{2}
$$

Again, $P_{x}$ is not a constant of the motion. One can anticipate that, for the system prepared in a non-stationary state of the complete Hamiltonian, the trap provides an acceleration mechanism, which changes the mean value of $P_{x}$, and, consequently, the splitting between spin states. In practice, the initial out-of-equilibrium situation was realized through the gravitational acceleration, i.e., via the scheme analyzed in the previous section: the system was prepared in a Gaussian superposition of momentum states whose center, $k_{x, i}$, was arranged to have a large-magnitude value, see Eq. (16). We intend to characterize the evolution followed by that state when the trap is connected. We will evaluate the conditions required for driving the momentum to the range of values where a transition between spin states becomes feasible. Moreover, we will identify the origin of the parallelism observed with the transitions induced by the gravitational scheme.

\section{A. Description of the inter-band transitions through the eikonal approximation}

As opposed to the case of the gravitational field, a complete analytical treatment of the system dynamics is not possible for a harmonic force. Still, some approximations can be implemented to analytically trace the applicability of the LZ model. Convenient for this aim are some considerations on the classical dynamics of the motional variables in the trap. Previous to the transition and provided that the coupling effects are neglected outside the crossing, the evolution of the system corresponds to a harmonic oscillator. Hence, the classical trajectory is characterized by a sinusoidal variation of both the coordinate and the momentum. From this classical picture, one can predict that, for the initial conditions corresponding to the considered out-of-equilibrium situation, the momentum eventually reaches the (reduced) value necessary for a crossing between spin states to take place. We recall that the momentum at the crossing, $k_{x, c}$, is given by Eq. (6); in particular, as previously pointed out, one finds $k_{x, c}=0$ for zero detuning. The required significant reduction in momentum implies that the initial energy has been converted, in large extent, into potential energy at 
the crossing. Additionally, we can expect that, in the analysis of the quantum dynamics, in order to put the focus on the transition region, a unitary transformation that incorporates a spatial translation to the crossing must be appropriate. Accordingly, we apply

$$
U=\exp \left[-\frac{i}{\hbar} q_{0} P_{x}\right]
$$

where the (static) displacement $q_{0}$ is chosen as the magnitude of the position at the crossing, i.e., of the coordinate corresponding to $k_{x}=k_{x, c}$. Following the previous considerations on the classical dynamics, we evaluate $q_{0}$ through the energy conservation

$$
q_{0}=\sqrt{\frac{2}{m \omega_{x}^{2}}}\left[\left\langle\frac{P_{x}^{2}}{2 m}+\frac{\alpha_{0} P_{x}}{\hbar}+\frac{1}{2} m \omega_{x}^{2} X^{2}\right\rangle_{\text {initial }}-\left(\frac{\left(\hbar k_{x, c}\right)^{2}}{2 m}+\alpha_{0} k_{x, c}\right)\right]^{1 / 2}
$$

where \langle\rangle$_{\text {initial }}$ denotes an average over the initial state. Note that $q_{0}$ increases with the energy. The transformed Hamiltonian is given by

$$
H_{T}^{\prime}=U^{\dagger} H_{T} U=\frac{P_{x}^{2}}{2 m}+\frac{1}{2} m \omega_{x}^{2}\left(X+q_{0}\right)^{2}+\frac{\hbar \Omega_{0}}{2} \sigma_{x}+\left(\frac{\hbar \delta_{0}}{2}+\frac{\alpha_{0} P_{x}}{\hbar}\right) \sigma_{z},
$$

where, the term $\frac{1}{2} m \omega_{x}^{2} X^{2}$ can be considered to be much smaller than $m \omega_{x}^{2} q_{0} X$ for a large enough displacement $q_{0}$, i.e., for an initial preparation corresponding to a sufficiently high energy. Then, $H_{T}^{\prime}$ can be approximated as

$$
H_{T}^{\prime}=\frac{P_{x}^{2}}{2 m}+m \omega_{x}^{2} q_{0} X+\frac{\hbar \Omega_{0}}{2} \sigma_{x}+\left(\frac{\hbar \delta_{0}}{2}+\frac{\alpha_{0} P_{x}}{\hbar}\right) \sigma_{z},
$$

where we have shifted the energy origin by $\frac{1}{2} m \omega_{x}^{2} q_{0}^{2}$. We emphasize that the restriction $\left\langle\frac{1}{2} m \omega_{x}^{2} X^{2}\right\rangle \ll\left\langle m \omega_{x}^{2} q_{0} X\right\rangle$, required by the validity of our derivation is guaranteed by the fulfillment of Eq. (17) for both, initial and final, momentum values. This is straightforwardly shown using the value of $q_{0}$, given by Eq. (21) and estimating the values of $\langle X\rangle$ in the crossing region, e.g. via energy conservation. It is important to stress that no additional limitations are being introduced in our framework.

Our approximate description of the system can be regarded as resulting from the application of the eikonal approximation [15]. Namely, in our procedure, the use of the unitary transformation that incorporates the displacement to the crossing point is equivalent to propose an ansatz for the complete wave function in terms of the product of a phase term and a reduced wave function in the momentum representation as the product of a highly oscillatory phase and a reduced smooth wave function. Specifically, we employ a phase 
linearly dependent on the momentum with a slope to be determined. Then, the optimum value of the extracted phase is obtained by requiring it to simplify the reduced Schrödinger equation. The applied simplification of the Hamiltonian strongly relies on the elimination of the term of harmonic confinement. For it to be sound, a large magnitude for the displacement is required. Moreover, the validity of the approach is improved with the accuracy of the translation to the crossing, i.e., with the precision in the determination of $q_{0}$. A more elaborate derivation of $q_{0}$, in a complete quantum formalism which incorporates details of the practical implementation, will be presented further on. The simple procedure followed by now is intended to stress the way in which the energy-dependent extracted phase affects the reduced Hamiltonian, and, in turn, as we shall see, the role of the energy of the initially prepared state in the characterization of the effective LZ parameters.

It is apparent that the Hamiltonian in Eq. (23) has the same form as that of the gravitational acceleration, given by Eq. (17). Here, the term $m \omega_{x}^{2} q_{0} X$ is the counterpart of the gravitational potential $H_{\text {grav }}=m g X$. The emergence of the LZ model in this scenario can be directly traced, as in the previous section, by consecutively applying two unitary transformations similar to those given by Eqs. (9) and (12). Accordingly, and, after the reduction to the spin space, we find

$$
H_{T}^{\prime \prime}=\frac{\hbar \Omega}{2} \sigma_{x}+\left[\frac{\hbar \delta_{0}}{2}+\frac{\alpha_{0}}{\hbar}\left(\hbar k_{x}-m \omega_{x}^{2} q_{0} t\right)\right] \sigma_{z} .
$$

Now, the comparison with Eq. (3) leads to the identification of the LZ characteristic parameters as:

$$
\begin{aligned}
& \zeta \rightarrow \frac{\Omega}{2} \\
& v \rightarrow-\frac{2 \alpha_{0} m \omega_{x}^{2} q_{0}}{\hbar^{2}}
\end{aligned}
$$

As in the gravitational scheme, the coupling strength is given by the offset. Common to the two considered acceleration schemes is also the proportionality existent between the effective splitting-variation rate $v$ and the SOC strength $\alpha_{0}$. Yet, there is a differential effect: in the harmonic setup, as opposed to the behavior found in the gravitational scheme, $v$ depends on the characteristics of the system preparation, in particular, on the energy. That dependence is incorporated in Eq. (25) through the trap frequency $\omega_{x}$ and the displacement to the crossing $q_{0}$. The magnitude of $v$ increases with $q_{0}$, and, therefore, with the energy 
of the system. Figure 1 illustrates the analogies and differences between the two considered acceleration schemes. The used parameters are similar to those corresponding to the experimental realization [10].

The simplified picture of the dynamics given by our approach uncovers the components of the system that are actually relevant to the LZ transition. It is the rate of variation of the momentum at the intersection point that determines the probability of switching between spin states. In turn, that rate is fixed by the energy of the initial preparation as far as transitions outside the crossing region can be neglected. As in the gravitational-acceleration scheme, it is found that the detuning does not affect the transition probability. Importantly, for our derivation to be valid, the preparation of the system must correspond to a sufficiently high energy. There are limitations on the energy values that can be achieved in the practical arrangement. Still, let us remind again that the conditions contained in Eq. (17) are safely satisfied by the experiments, which explains the robustness of the LZ approach: despite the variety of used parameters, the model was found to accurately reproduce the observed features. It is important to point out that, in our analysis, as in the experiments, only one crossing is being considered. The periodic character of the dynamics of the external variables in the harmonic trap is not tackled.

\section{B. A model for the dynamics outside the crossing}

In the former analysis, the focus was put on the transition region. The optimum displacement $q_{0}$ used in the unitary transformation given by Eq. (20) was evaluated through simple classical arguments on energy conservation. Let us present now a more complete (quantum) picture of the dynamics previous to the crossing which incorporates specific aspects of the experimental realization. This framework will provide us with another method for deriving $q_{0}$.

In order to characterize the evolution of the diabatic states, we can reasonably assume, as previously, that the coupling, accounted for by the offset term in the Hamiltonian, becomes

effective only very near the crossing. Hence, as the transition is approached, the evolution of the initially prepared state can be regarded as given by the Hamiltonian in Eq. (18) where the offset is neglected in the term $H_{S O}$. The resulting Hamiltonian becomes spin-separable, and, for each spin value, it corresponds to a displaced harmonic oscillator. Namely, the 
dynamics before the crossing can be assumed to be governed by

$$
H_{T, \pm}=\hbar \omega_{x} a^{\dagger} a \pm\left(\frac{\hbar \delta_{0}}{2}+\frac{\alpha_{0}}{\hbar} i \sqrt{\frac{m \hbar \omega_{x}}{2}}\left(a^{\dagger}-a\right)\right),
$$

where \pm refers to the spin components, and, $a^{\dagger}$ and $a$ are the creation and annihilation operators associated to the $x$-coordinate.

The procedure to obtain the eigenstates of $H_{T, \pm}$ is straightforward. Let us illustrate here its application to $H_{T,+}$. First, we use the unitary transformation given by

$$
U=D(\beta)
$$

where $D(\beta)$ is the displacement operator [14] with argument $\beta$ to be determined by imposing the intended reduction of the description. The transformed Hamiltonian is given by

$$
H_{T,+}^{\prime}=D^{\dagger}(\beta) H_{T,+} D(\beta)=\hbar \omega_{x}\left(a^{\dagger}+\beta^{\star}\right)(a+\beta)+\frac{\hbar \delta_{0}}{2}+\frac{\alpha_{0}}{\hbar} i \sqrt{\frac{m \hbar \omega_{x}}{2}}\left(a^{\dagger}+\beta^{\star}-a-\beta\right) .
$$

Now, by choosing

$$
\beta=-i \frac{\alpha_{0}}{\hbar} \sqrt{\frac{m}{2 \hbar \omega_{x}}},
$$

$H_{T,+}^{\prime}$ is converted into the Hamiltonian of an undisplaced harmonic oscillator, namely, into

$$
H_{T,+}^{\prime}=\hbar \omega_{x} a^{\dagger} a+\hbar \omega_{x}|\beta|^{2}-\frac{\alpha_{0}^{2}}{\hbar^{2}} m
$$

where the constant terms simply shift the energy origin. Important for the comparison with the former derivation of $q_{0}$ is to take into account that the applied unitary transformation implies working with a displaced momentum, the associated operator $\tilde{P}_{x}=D^{\dagger}(\beta) P_{x} D(\beta)$ being given by

$$
\tilde{P}_{x}=P_{x}+\sqrt{2 m \hbar \omega_{x}} \operatorname{Im}(\beta)=P_{x}+\frac{\alpha_{0}}{\hbar} m .
$$

It is pertinent to refer here to the role of the gravitational acceleration in the current scheme, where the harmonic trap is connected. Actually, the gravitational potential can be included in our framework through a unitary transformation that displaces the harmonic oscillator in the vertical coordinate. Therefore, it does not introduce qualitative differences in the dynamics: it simply modifies the equilibrium position of the trapping potential. 
In the representation of eigenstates of $H_{T+}^{\prime}$, the evolution of the initially prepared state is direct. This is the case, in particular, if we assume that the initial preparation corresponds to a coherent state, $|\eta\rangle$, i.e., to an eigenstate of the annihilation operator, $a|\eta\rangle=\eta|\eta\rangle$, where $\eta$ is the complex number from which all the state characteristics are determined. Hence, we take for the initial state

$$
|\varphi(t=0)\rangle=|\eta\rangle
$$

whose associated wave function is given by [16]

$$
\varphi(x, t=0)=\langle x \mid \eta\rangle=e^{i \theta_{\eta}}\left(\frac{m \omega_{x}}{\pi \hbar}\right)^{1 / 4} \exp \left\{-\left[\frac{x-\langle X\rangle_{\eta}}{2 \Delta X_{\eta}}\right]^{2}+i\langle P\rangle_{\eta} \frac{x}{\hbar}\right\}
$$

with

$$
\begin{aligned}
\langle X\rangle_{\eta} & =\sqrt{\frac{2 \hbar}{m \omega_{x}}} \operatorname{Re}(\eta), \\
\left\langle P_{x}\right\rangle_{\eta} & =\sqrt{2 m \hbar \omega_{x}} \operatorname{Im}(\eta), \\
\Delta X_{\eta} & =\sqrt{\frac{\hbar}{2 m \omega_{x}}},
\end{aligned}
$$

and where the global phase $\theta_{\eta}$ plays no physical role. By Fourier transforming $\varphi(x, t=0)$, a parallel form for the counterpart function in the momentum representation is obtained. The momentum distribution of a coherent state simulates the initial preparation given by Eq. (16). In fact, for the parallelism to be complete, the width parameter $\sigma_{w}$ of the practical realization must match that of a minimum-uncertainty wave packet. However, we will see that our conclusions are robust when the width of the Gaussian is varied. As it is relevant also to the analysis of many-body effects, this issue will be dealt with in the next section. By now, we assume that the preparation can be modeled by a coherent state and that $\eta$ is known from the experimental conditions.

Switching to the new representation $|\tilde{\varphi}\rangle$, we have $|\tilde{\varphi}(t=0)\rangle=D^{\dagger}(\beta)|\varphi(t=0)\rangle=$ $D(-\beta)|\varphi(t=0)\rangle$. Moreover, taking into account that the initial state can be expressed as a displaced vacuum [14], i.e., $|\varphi(t=0)\rangle=D(\eta)|0\rangle$, we can write

$$
|\tilde{\varphi}(t=0)\rangle=D(-\beta) D(\eta)|0\rangle=e^{i \operatorname{Im}\left(-\beta \eta^{\star}\right)} D(\eta-\beta)|0\rangle=e^{i \operatorname{Im}\left(-\beta \eta^{\star}\right)}|\eta-\beta\rangle .
$$

Hence, the initial state corresponds to the coherent state $|\eta-\beta\rangle$ of the transformed Hamiltonian. Its time evolution is given by [14] 


$$
|\tilde{\varphi}(t)\rangle=e^{i \operatorname{Im}\left(-\beta \eta^{\star}\right)}\left|(\eta-\beta) e^{-i \omega_{x} t}\right\rangle
$$

In particular, at the crossing, $\left(t=t_{c}\right)$, we have for the mean values of the coordinate and (displaced) momentum

$$
\begin{aligned}
q_{0} & =\sqrt{\frac{2 \hbar}{m \omega_{x}}} \operatorname{Re}\left[(\eta-\beta) e^{-i \omega_{x} t_{c}}\right], \\
\hbar k_{x, c}+\frac{\alpha_{0}}{\hbar} m & =\sqrt{2 m \hbar \omega_{x}} \operatorname{Im}\left[(\eta-\beta) e^{-i \omega_{x} t_{c}}\right]
\end{aligned}
$$

where we have made use of the applicability of Eqs. (34) and (35) to any coherent state. Combining the above equations, the optimum displacement to introduce in the unitary transformation in Eq. (20) is evaluated as

$$
q_{0}=\sqrt{\frac{2}{m \omega_{x}^{2}}}\left[\hbar \omega_{x}|\eta|^{2}+\frac{\alpha_{0}}{\hbar} \sqrt{2 m \hbar \omega_{x}} \operatorname{Im}(\eta)-\left(\frac{\left(\hbar k_{x, c}\right)^{2}}{2 m}+\alpha_{0} k_{x, c}\right)\right]^{1 / 2}
$$

Moreover, taking into account that

$$
\left\langle\eta\left|\frac{P_{x}^{2}}{2 m}+\frac{\alpha_{0} P_{x}}{\hbar}+\frac{1}{2} m \omega_{x}^{2} X^{2}\right| \eta\right\rangle=\hbar \omega_{x}|\eta|^{2}+\frac{\alpha_{0}}{\hbar} \sqrt{2 m \hbar \omega_{x}} \operatorname{Im}(\eta),
$$

we recover the value of $q_{0}$ given by Eq. (21). The complete agreement between the two presented derivations of $q_{0}$ is rooted in the quasi-classical character of the evolution of a coherent state and in the crucial role played by the trajectory of the center of the Gaussian wave packet in the effective model. That correspondence is a proof of the validity of our simple approach. Again, the analogy of our procedure with the eikonal approximation is apparent: the application of the unitary transformation given by Eq. (20) is equivalent to extract the phase of the coherent-state wave function in the momentum representation. Namely, the term $\exp \left[-\frac{i}{\hbar}\langle X\rangle_{\eta} p\right]$ is taken out as a factor, and, the resulting reduced Hamiltonian is cast into the LZ scenario.

It is of interest to discuss at this point the possibility of reaching the adiabatic regime, i.e., of significantly diminishing $P_{L Z}^{(a)}=e^{-2 \pi|\zeta|^{2} /|v|}$. A reduction in $v$ can only be obtained via the decrease of $q_{0}$, see Eq. (25). However, as previously stated, a significant value of $q_{0}$ is needed for the consistency of our approach. Then, reducing $v$ is not allowed, and, in order to diminish the probability of transition between adiabatic states, one can only 
think of increasing the offset, as found in the case of gravitational acceleration. However, the implementation of this requirement, extracted from an approximate description of the system which involves the translation to the crossing, can introduce inconsistencies in our framework. Actually, a large offset can induce transitions previous to the crossing, and, consequently, invalidate the implemented approximation. Anyway, in that case, i.e., for a sufficiently large value of $\Omega$, a useful alternative strategy to tackle the dynamics is the application of an adiabatic approximation in the original Hamiltonian, with no simplification. That procedure is more complex than the use of the translation technique, but it is still feasible.

\section{MANY-BODY EFFECTS}

The description of many-body interaction effects is required by the considered practical conditions, which corresponded to the preparation as a condensate. In a preliminary analysis

of the experiments [10], the role of interaction effects in the appearance of the observed features was evaluated. Given that, in the implemented setup, the magnitude of the atomicinteraction energy was much smaller than the kinetic energy, a single-particle approach was considered to be sufficiently accurate to describe the dynamics. The agreement between the predictions of the (single-particle) LZ model and the observed features validates that conclusion. Here, we intend to go beyond the conditions corresponding to the particular experimental realization and assess the robustness of the single-particle approach as the magnitude of the interaction is increased. The clarification of this aspect of the dynamics is crucial for evaluating the potential applicability of the studied transitions in different contexts, and, in particular, for assessing their usefulness in strategies of control. In our analysis, we proceed by reviewing first some general characteristics of the interaction. Then, we present a quantitative evaluation of the corrections that many-body effects introduce in the single-particle approach. In this way, we delimit the range of safe applicability of the basic LZ scenario. 


\section{A. General characterization of the interaction effects}

From the knowledge of the system dynamics provided by the present study and by previous related work, the following general arguments can be outlined:

Due to the SOC, the system presents specific atomic-interaction characteristics. In particular, there are different interaction strengths associated with the spin combinations. They will be denoted as $g_{\eta, \eta^{\prime}}$, where $\eta, \eta^{\prime}=1,2$ refer to the spins. As shown in previous studies, depending on the SOC parameters and on the values of $g_{\eta, \eta^{\prime}}$, the form of the wave-function of the ground state can display a varied topology [17]. In the case of uniform confinement, three phases, known as stripe, plane-wave (separate dressed-state), and single-minimum phases, can appear [17, 18]. (See also [19] for experimental studies of finite-temperature phase diagrams). These phases persist when harmonic confinement is considered. Apart from altering the form of the ground state, many-body effects can modify the evolution of the initially prepared state. Both aspects are relevant to the considered setup. Their detailed description is quite complex in a general regime. Still, the identification of some of their general characteristics can be sufficient to evaluate their role in the present context. As in the preliminary analysis of the experiments, we assume here that the realized initial state is satisfactorily simulated by the product of one of the spin states and an external state with a Gaussian distribution of momenta. We aim at identifying differential aspects in the evolution of this state associated with the inclusion of many-body effects.

Important for our discussion is to recall some results of former work on the application of a variational method to characterize the evolution of a Bose-Einstein condensate. In the absence of spin-orbit coupling, the use of a Gaussian ansatz with a set of dynamical variational parameters, referring to the center and to the widths of the Gaussian, showed that the evolution of the center is uncoupled from the dynamics of the rest of the parameters [20]. Moreover, the atomic interaction was found to play no role in the dynamics of the center, which presented no differences with that given by a single-particle harmonic Hamiltonian. Many-body effects alter the dynamics of the other parameters, i.e., the form of the Gaussian, and, consequently, the distribution of momenta. These results, explicitly obtained for a harmonic confinement, can be shown to be valid also for a linear potential, and, therefore, for the case of gravitational acceleration.

In recent studies [21], the same variational technique has been applied to analyze the 
evolution of spin-orbit coupled Bose-Einstein condensates in the regime of separate dressed states. The conclusions on the lack of coupling between the evolution of the center of the Gaussian and the dynamics of other modes still apply provided that the different interaction strengths relevant to the SOC system do not significantly differ, specifically, when $g_{2} \equiv$ $\left|\left(g_{12}-g_{11}\right) / 4\right| \ll g_{1} \equiv\left(g_{12}+g_{11}\right) / 4,\left(g_{11} \simeq g_{22}\right)$. This restriction is fulfilled for the systems analyzed in standard experimental realizations. As $g_{2}$ increases, the evolution of the center becomes coupled with the dynamics of other modes; moreover, nonlinear effects set in.

\section{B. Evaluation of the corrections to the single-particle description}

We turn now to set up a framework where a quantitative analysis of many-body effects can be carried out. The starting point is the time-dependent Gross-Pitaevskii equation

$$
i \hbar \frac{\partial \mathbf{\Psi}_{\mathbf{s}}(\mathbf{r}, t)}{\partial t}=\left[H+N g_{\text {int }} \rho(\mathbf{r}, t)\right] \mathbf{\Psi}_{\mathbf{s}}(\mathbf{r}, t)
$$

where $H=-\frac{\hbar^{2}}{2 m} \nabla_{\mathbf{r}}^{2}+V_{e x}(\mathbf{r})+\frac{\hbar \Omega}{2} \sigma_{x}+\left(\frac{\hbar \delta_{0}}{2}-i \alpha_{0} \frac{\partial}{\partial x}\right) \sigma_{z}$ with $V_{e x}(\mathbf{r})=\frac{1}{2} m\left(\omega_{x}^{2} x^{2}+\omega_{y}^{2} y^{2}+\omega_{z}^{2} z^{2}\right)$,

and $\Psi_{\mathbf{s}} \equiv\left(\begin{array}{c}\Psi_{+}(\mathbf{r}, t) \\ \Psi_{-}(\mathbf{r}, t)\end{array}\right)$ denotes a two-component spinor wave function in the coordinate representation, $N$ is the number of particles, and $\rho(\mathbf{r}, t)=\left|\Psi_{+}\right|^{2}+\left|\Psi_{-}\right|^{2}$. Note that a threedimensional description is necessary. For simplicity, we consider that the involved interaction strengths are equal, specifically, $g_{i n t} \equiv g_{11}=g_{22}=g_{12}$. The implications of dealing with different scattering lengths will be discussed. In our procedure to solve Eq. (43), we apply first a variational method in the line of previous studies on SOC condensates. Despite the restrictions associated with the lack of generality of the proposed ansatz, the results will provide us with useful clues to setting up a scheme of general validity. From that approach, the predictions of the single-particle study will be recovered.

\section{The variational approach}

Our method combines elements of previous applications of variational techniques to related systems. First, we propose an ansatz for the evolution of the center of the packet via the sequence of unitary transformations 


$$
\Psi_{\mathbf{s}}(\mathbf{r}, t)=U_{4}(t) U_{3}(t) \Psi(\mathbf{r}, t)
$$

where

$$
\begin{aligned}
& U_{3}(t)=\exp \left[-\frac{i}{\hbar} \mathbf{r}_{c}(t) \mathbf{P}\right], \\
& U_{4}(t)=\exp \left[-\frac{i}{\hbar} \mathbf{p}_{c}(t) \mathbf{R}\right],
\end{aligned}
$$

with $\mathbf{P}$ and $\mathbf{R}$ being the particle position and momentum vector operators. For the transformed wave function, we propose the ansatz

$$
\boldsymbol{\Psi}(\mathbf{r}, t)=\Phi_{0}(\mathbf{r}, t)\left(\begin{array}{c}
c_{+}(t) \\
c_{-}(t)
\end{array}\right)
$$

Note that, as no spatial dependence is allowed for the coefficients $c_{+}$and $c_{-}$, the proposal lacks generality. Still, as opposed to former applications of the variational techniques [21], no restrictions are imposed on the time dependence of the spin coefficients and on the functional form of $\Phi_{0}(\mathbf{r}, t)$. We shall now determine the functions $\mathbf{r}_{c}(t), \mathbf{p}_{c}(t), \Phi_{0}(\mathbf{r}, t), c_{+}(t)$ and $c_{-}(t)$ from the stationary action principle

$$
\delta \int_{t_{0}}^{t} d t^{\prime} \int d^{3} \mathbf{r} \Psi_{\mathbf{s}}^{\dagger}\left[H-i \hbar \frac{\partial}{\partial t^{\prime}}+\frac{1}{2} N g_{i n t} \rho\left(\mathbf{r}, t^{\prime}\right)\right] \Psi_{\mathbf{s}}=0
$$

where $\Psi_{\mathrm{s}}^{\dagger} \equiv\left(\Psi_{+}^{\star}, \Psi_{-}^{\star}\right)$ is the transpose complex conjugate spinor wave function. The solution must satisfy the supposedly initial known form $\mathbf{\Psi}_{\mathbf{s}}\left(\mathbf{r}, t_{0}\right)$ of this spinor wavefunction. If $\mathbf{r}_{c}\left(t_{0}\right)$ and $\mathbf{p}_{c}\left(t_{0}\right)$ are chosen such that

$$
\begin{aligned}
& \left\langle\Phi_{0}\left(t_{0}\right)|\mathbf{R}| \Phi_{0}\left(t_{0}\right)\right\rangle=\mathbf{0} \\
& \left\langle\Phi_{0}\left(t_{0}\right)|\mathbf{P}| \Phi_{0}\left(t_{0}\right)\right\rangle=\mathbf{0} .
\end{aligned}
$$

the variational solutions for $\mathbf{r}_{c}(t)$ and $\mathbf{p}_{c}(t)$, intended to define the trajectory of the packet center, are given by the set of equations 


$$
\begin{aligned}
\left(\begin{array}{c}
\dot{x}_{c} \\
\dot{y}_{c} \\
\dot{z}_{c}
\end{array}\right) & =\frac{1}{m}\left(\begin{array}{l}
p_{c, x} \\
p_{c, y} \\
p_{c, z}
\end{array}\right)+\left[\left|c_{+}\right|^{2}-\left|c_{-}\right|^{2}\right] \frac{\alpha_{0}}{\hbar}\left(\begin{array}{l}
1 \\
0 \\
0
\end{array}\right), \\
\left(\begin{array}{c}
\dot{p}_{c, x} \\
\dot{p}_{c, y} \\
\dot{p}_{c, z}
\end{array}\right) & =-m\left(\begin{array}{l}
\omega_{x}^{2} x_{c} \\
\omega_{y}^{2} y_{c} \\
\omega_{z}^{2} z_{c}
\end{array}\right),
\end{aligned}
$$

The variational solution for $\Phi_{0}(\mathbf{r}, t)$ is provided by the equation

$$
i \hbar \frac{\partial \Phi_{0}}{\partial t}=\left[E_{c}-\dot{\mathbf{r}}_{c} \mathbf{p}_{c}-\frac{\hbar^{2}}{2 m} \nabla_{\mathbf{r}}^{2}+V_{e x}+N g_{i n t}\left|\Phi_{0}\right|^{2}\right] \Phi_{0},
$$

with $E_{c}(t)=\frac{1}{2 m} \mathbf{p}_{c}^{2}+\frac{1}{2} m\left(\omega_{x}^{2} x_{c}^{2}+\omega_{y}^{2} y_{c}^{2}+\omega_{z}^{2} z_{c}^{2}\right)+\left(\left|c_{+}\right|^{2}-\left|c_{-}\right|^{2}\right) \frac{\alpha_{0}}{\hbar} p_{c, x}$. Finally, the evolution of the spin coefficients $c_{+}(t)$ and $c_{-}(t)$ is found to be given by

$$
i \hbar \frac{\partial}{\partial t}\left(\begin{array}{l}
c_{+} \\
c_{-}
\end{array}\right)=\left[\frac{\hbar \Omega}{2} \sigma_{x}+\left(\frac{\hbar \delta_{0}}{2}+\frac{\alpha_{0}}{\hbar} p_{c, x}\right) \sigma_{z}\right]\left(\begin{array}{l}
c_{+} \\
c_{-}
\end{array}\right) .
$$

Hence, the application of the variational method with an ansatz with spatial-independent coefficients $c_{+}$and $c_{-}$leads exactly to the LZ scenario. It is worth emphasizing that the functions $\mathbf{r}_{c}(t)$ and $\mathbf{p}_{c}(t)$ [see Eqs. (50) and (51)], describe the dynamics of a classical harmonic oscillator modified by a spin-dependent driving term. Additionally, the dynamics of $\Phi_{0}$ parallels that of the wave function of a condensate in the absence of SOC in a harmonic trap. Indeed, Eq. (52) can be cast into the standard form of the time-dependent GrossPitaevskii equation by incorporating the extra (time-dependent, coordinate-independent terms $) E_{c}$ and $\dot{\mathbf{r}}_{c} \mathbf{p}_{c}$ as a phase $\Phi_{0}(\mathbf{r}, t) \rightarrow \Phi_{0}(\mathbf{r}, t) e^{-\frac{i}{\hbar} \int_{t_{0}}^{t}\left(E_{c}-\dot{\mathbf{r}}_{c} \mathbf{p}_{c}\right) d t^{\prime}}$. In order to simplify, the discussion of our results, the formal elimination of those terms will be considered in the following. Hence, without changing the notation for the transformed wave function, we rewrite Eq. (52) as

$$
i \hbar \frac{\partial \Phi_{0}}{\partial t}=\left[-\frac{\hbar^{2}}{2 m} \nabla_{\mathbf{r}}^{2}+V_{e x}+N g_{i n t}\left|\Phi_{0}\right|^{2}\right] \Phi_{0}
$$




\section{Generalization of the method: results for an ansatz with general validity}

Now, taking as starting point the scheme provided by the variational method, we turn to set up a framework of general validity. Again, we use the sequence of unitary transformations given by Eqs. (44), (45), (46), but, now, a generalized proposal is made for the trajectory of the packet center: although Eq. (51) still applies, Eq. (50) is modified as

$$
\left(\begin{array}{c}
\dot{x}_{c} \\
\dot{y}_{c} \\
\dot{z}_{c}
\end{array}\right)=\frac{1}{m}\left(\begin{array}{l}
p_{c, x} \\
p_{c, y} \\
p_{c, z}
\end{array}\right)+\left[\left\langle\left|c_{+}\right|^{2}\right\rangle-\left\langle\left|c_{-}\right|^{2}\right\rangle\right] \frac{\alpha_{0}}{\hbar}\left(\begin{array}{l}
1 \\
0 \\
0
\end{array}\right),
$$

with $c_{+}(\mathbf{r}, t)$ and $c_{-}(\mathbf{r}, t)$ being functions, with spatial dependence, that we use in a different (general) characterization of the spinor wave function: without loss of generality, we write

$$
\boldsymbol{\Psi}(\mathbf{r}, t)=\Phi_{0}(\mathbf{r}, t)\left(\begin{array}{l}
c_{+}(\mathbf{r}, t) \\
c_{-}(\mathbf{r}, t)
\end{array}\right) .
$$

The function $\Phi_{0}(\mathbf{r}, t)$ is now imposed to obey Eq. (52), which does not imply a restriction

since $c_{+}$and $c_{-}$incorporate spatial dependence. Note that $\left\langle\left|c_{ \pm}\right|^{2}\right\rangle \equiv\left\langle\left.\Phi_{0}|| c_{ \pm}\right|^{2} \mid \Phi_{0}\right\rangle$. Then, through the application of the sequence of unitary transformations and the use of our ansatz for the transformed wave function in Eq. (43), the evolution of the spin coefficients is found to be given by

$$
\begin{aligned}
i \hbar \frac{\partial}{\partial t}\left(\begin{array}{c}
c_{+} \\
c_{-}
\end{array}\right)= & \left\{-\frac{\hbar^{2}}{2 m} \nabla_{\mathbf{r}}^{2}-\frac{\hbar^{2}}{m \Phi_{0}}\left(\nabla_{\mathbf{r}} \Phi_{0}\right) \nabla_{\mathbf{r}}+N g_{i n t}\left|\Phi_{0}\right|^{2}\left(\left|c_{+}\right|^{2}+\left|c_{-}\right|^{2}-1\right)+\right. \\
& -\frac{i \alpha_{0}}{\Phi_{0}} \frac{\partial \Phi_{0}}{\partial x}\left[\sigma_{z}-\left(\left\langle\left|c_{+}\right|^{2}\right\rangle-\left\langle\left|c_{-}\right|^{2}\right\rangle\right)\right]-i \alpha_{0} \sigma_{z} \frac{\partial}{\partial x}+ \\
& \left.\frac{\hbar \Omega}{2} \sigma_{x}+\left(\frac{\hbar \delta_{0}}{2}+\frac{\alpha_{0}}{\hbar} p_{c, x}\right) \sigma_{z}\right\}\left(\begin{array}{l}
c_{+} \\
c_{-}
\end{array}\right) .
\end{aligned}
$$

Note that, in setting up our framework, no approximations have been made apart from the mean-field approach that leads to the Gross-Pitaevskii equation. Despite the complexity of the description, the following basic characteristics of the role of many-body effects in the emergence of the LZ scenario can be identified: 
i) Interaction effects enter directly Eq. (54): they affect the form of $\Phi_{0}$. In turn, as $\Phi_{0}$ varies, the dynamics of the spin coefficients, given by Eq. (57), is modified. The indirect role of many-body effects in the trajectory of the packet center is also apparent. Moreover, since Eq. (54) has the same functional structure as that corresponding to the dynamics of a condensate in the absence of SOC, a parallelism can be traced between both systems. This implies the potential utility in our system of the well-known characterization of the condensate without SOC.

ii) The departure of Eq. (57) from the standard LZ scenario is rooted in the terms that incorporate spatial derivatives, which are actually relevant when the functions $c_{+}$and $c_{-}$ are nonuniform, and in the term that includes $\left|\Phi_{0}\right|^{2}$. As the preparation of the system in the experiments can be approximated by the product of one of the spin states and the fundamental state of a condensate in a harmonic trap, $c_{+}$and $c_{-}$are initially uniform. The spatial derivatives are activated only when, due to the coupling term $\frac{\hbar \Omega}{2} \sigma_{x}$, non-uniformity sets in. Specifically, the role of non-uniformity initiates when the transfer of population modifies the initial value of the difference $\left\langle\left|c_{+}\right|^{2}\right\rangle-\left\langle\left|c_{-}\right|^{2}\right\rangle$, which is 1 or -1 , depending on the prepared spin state. The same argument applies to the term with $\left|\Phi_{0}\right|^{2}$. Consequently, we conclude that it is only near the crossing that the form of $\Phi_{0}$, in particular, its evolution, due to interaction effects, becomes relevant to the dynamics of the spin functions.

iii) Let us see that the form of the expressions obtained for the probability of transition in the single-particle approach is robust against many-body effects. Important to this point is to recall that the nonuniform terms that affect the evolution of the spin functions are also present in the single-particle description, which can be simply recovered by taking $g_{\text {int }}=0$ in Eq. (54) and Eq. (57). Spatial variation of the wave function, given then by the Schrödinger equation, enters the corrections to the basic LZ model. Even when the (position-dependent) fundamental state of the harmonic trap is prepared and no changes take place in $\Phi_{0}$, spatial derivatives are activated as soon as the transfer of population becomes relevant. As previously discussed, those corrections are small provided that the coupling becomes effective only very near the crossing, which is in turn guaranteed by the asymptotic character of the LZ scenario. The order of magnitude of the corrections does not significantly varies when interaction is considered, and, therefore, the form of the LZ formula is robust. It is clear that these conclusions give validity to our use, in the previous section, of a coherent state in the simulation of the single-particle dynamics irrespective of 
the actual value of the Gaussian width.

Our framework can be easily adapted to deal with the case of gravitational acceleration. Indeed, it is shown that the result referring to the exact validity of the LZ model for gravitational acceleration in the single-particle case can be recovered. Switching to the many-body scenario, one can conjecture that, due to the lack of confinement in the gravitational acceleration, the (repulsive) interaction effects must be less important that in the case of harmonic trapping: the unimpeded spreading of the wave function implies smaller spatial derivatives.

iv) The asymptotic character of the LZ predictions must be stressed: the LZ formula connects the initial and final configurations, which are both very far from the crossing. The compact form of the transition probability is actually due to these characteristics. It is again apparent that the magnitude of the coupling term $\frac{\hbar \Omega}{2} \sigma_{x}$ determines the definition of the asymptotic regions. As $\Omega$ increases, larger distances from the crossing are required for the initial and final configurations. This condition implies limitations on the practical realization. Here, it is worth mentioning the results of [22], where departures from the LZ predictions were observed for increasing values of $\Omega$; also in agreement with our results, deformation of the wave packet due to interaction effects were observed.

v) Our approach allows us to evaluate the effect of interactions on the evolution of the center of the wave packet. This point is crucial since it is the dynamics of the mean value of the momentum, i.e., the trajectory of the center of the packet, that determines the sweeping-rate parameter of the simplified description of the transitions. From Eqs. (51) and (55), one can conclude that, again, it is near the crossing, where interaction effects become effective, that the spin-induced driving of the trajectory can be significant. A modification of the LZ velocity can be expected, but, still, the applicability of the LZ formula for the probability of transition is guaranteed provided that the asymptotic conditions are fulfilled. The analysis of Eq. (55) shows that the relative importance of the spin-dependent driving of the center-of-the-packet variables decreases as the quotient between $\frac{\alpha_{0}}{\hbar}$ and the magnitude of $\frac{p_{c, x}}{m}$ diminishes. The experimental conditions [10] indeed correspond to a very small value of that quotient. Indeed, a perturbative treatment of the terms additional to the primary LZ scenario shows that it is only beyond first order that corrections to the spin population given by the basic LZ model appear. A detailed presentation of the technical details of that treatment will be given in future work.

From the above general picture of the dynamics, one can infer the sound applicability 
of the basic LZ model to the many-particle scenario in standard conditions. Departures from the above description appear when the magnitudes of the different scattering lengths (diagonal and non-diagonal) relevant to the problem are significantly different. It can be shown that then the spin functions $c_{+}$and $c_{-}$enter the equation for the dynamics of $\Phi_{0}(\mathbf{r}, t)$, i.e., Eq. (54) is significantly modified. Moreover, the different scattering lengths appear explicitly in the equation that gives the evolution of the spin functions. We intend to evaluate in future work if the basic structure of the LZ model can still be traced in this more complex scenario.

Finally, it is pertinent to stress the substantial differences between the system analyzed here and other variations of the LZ scenario, relevant to different contexts, which present a significant modification of the single-particle output. For instance, it is worth mentioning studies which have focused on the effect of a nonlinear coupling [23] and of a nonlinear sweeping rate [24]. There, the term nonlinear refers to dependence on the spin populations. As opposed to those models, in our system, no dependence of the coupling term (or of the sweeping rate) on the spin populations is present. Again, we stress that departures from this picture can be expected when different (spin-dependent) scattering lengths are considered.

\section{CONCLUDING REMARKS}

In our study, the LZ model has been generalized along two lines. First, we have enlarged the dimensionality of the system by including the external variables. This has been done for two forms of the external forces: linear and quadratic potentials have been considered. In both cases, the emergence of the basic LZ model has been analytically traced. Second, we have incorporated many-body effects. Given the characteristics of the atomicinteraction strengths, the many-body dynamics does not alter the applicability of the model: the single-particle description has been found to be robust when atomic-interaction effects are incorporated.

Our analysis has uncovered the crucial role played by the properties of the initial preparation of the system in the applicability of the LZ description. We have found that, for the LZ model to be valid, the momentum components of the initial state must correspond to large values of that observable. The measurement on the system must also be carried out when the momentum has reached again a large-magnitude value, i.e., the effective LZ ramp 
must be ended sufficiently far from the crossing. These requirements were fulfilled in the experimental realization.

The above arguments are also relevant to the observed robust character of the applicability of the model when the characteristics of the acceleration methods are varied. The use of different acceleration schemes only alters the dynamics outside the crossing, which is relevant merely to determine the rate of variation of the momentum at the transition. As a side effect of the validity of the LZ approach, the lack of dependence of the transition probability on the detuning has been explained. Limitations on reaching an adiabatic regime for the transitions have been revealed: only by increasing the offset parameter, the transition between adiabatic states can be inhibited in practice.

The exact analytical results obtained for the scheme based on gravitational acceleration can find additional applicability in the precise characterization of the evolution of different initial states outside the asymptotic regime. The use of the LZ approach is frequently limited to the evaluation of asymptotic probabilities of transition. From our analytical approach, a description that goes beyond that framework is feasible.

Apart from a more complete explanation of the experimental results, our analytical results can provide clues to steer the dynamics. The study gives additional support to the strategy of control proposed in Ref. [10]: the spin polarization of the output can be tuned using the offset and the rate of splitting variation as parameters of control. It is also apparent that the study can be relevant to strategies to manipulate the system based on the variation of the SOC parameters [7-9]. Indeed, it is pertinent to take into account the potential role of the trapping potential as a mechanism of acceleration whenever a non-equilibrium situation is tackled. The analysis can provide clues to advancing in the characterization of aspects of the dynamics of spin-orbit coupled Bose-Einstein condensates like the coupling of collective modes [17, 21, 25 27] and the differential effects of the diverse ground-state characteristics. 


\section{Acknowledgments}

One of us (JMGL) acknowledges the support from Ministerio de Economía y Competitividad (Grant No. FIS2013-41532-P) and from the European Regional Development Fund.

[1] Y.-J. Lin, K. Jiménez-García, and I. B. Spielman, Nature (London) 471, 83 (2011).

[2] I. B. Spielman, Phys. Rev. A 79, 063613 (2009).

[3] P. Wang, Z-Q. Yu, Z. Fu, J. Miao, L. Huang, S. Chai, H. Zhai, and J. Zhang, Phys. Rev. Lett. 109, 095301 (2012).

[4] L. W. Cheuk, A. T. Sommer, Z. Hadzibabic, T. Yefsah, W. S. Bakr, and M. W. Zwierlein, Phys. Rev. Lett. 109, 095302 (2012).

[5] N. Goldman, G. Juzeliunas, P. Ohberg, and I. B. Spielman, Rep. Prog. Phys. 77, 126401 (2014).

[6] J. Dalibard, F. Gerbier, G. Juzeliunas, and P. Ohberg, Rev. Mod. Phys. 83, 1523 (2011).

[7] K. Jiménez-García et al., Phys. Rev. Lett. 114, 125301 (2015). (See also the Supplemental Material).

[8] Y. Zhang, G. Chen, and C. Zhang, Sci. Rep. 3, 1937 (2013).

[9] J.M Gomez Llorente and J. Plata, Phys. Rev. A 93, 063633 (2016).

[10] A. J. Olson, Su-Ju Wang, R. J. Niffenegger, Chuan-Hsun Li, C. H. Greene, and Y. P. Chen, Phys. Rev. A 90, 013616 (2014).

[11] L. D. Landau, Phys. Z. Sowjetunion 2, 46 (1932).

[12] C. Zener, Proc. R. Soc. A 137, 696 (1932).

[13] B. P. Anderson and M. A. Kasevich, Science 282, 1686 (1998).

[14] W.H. Louisell, Quantum Statistical Properties of Radiation (John Wiley, New York, 1973).

[15] J. J. Sakurai, Modern Quantum Mechanics (Addison-Wesley Publishing Company, Inc., Reading, MA, 1994).

[16] C. Cohen-Tannoudji, B. Diu, and F. Laloe, Quantum Mechanics, (John Wiley, New York, 1977).

[17] Y. Li, G.I. Martone, and S. Stringari (2015) Spin-Orbit-coupled Bose-Einstein condensates. Annual Review of Cold Atoms and Molecules: pp. 201-250. 
[18] Tin-Lun Ho and Shizhong Zhang, Phys. Rev. Lett. 107, 150403 (2011).

[19] Jin-Yi Zhang, Long Zhang, Zhi-Dong Du, Wei Zheng, You-Jin Deng, Hui Zhai, Shuai Chen, Jian-Wei Pan, Nature Physics 10, 314 (2014).

[20] V.M. Perez-Garcia, H. Michinel, J.I. Cirac, M. Lewenstein, and P. Zoller, Phys. Rev. A 56, 1424 (1997).

[21] Z. Chen and H. Zhai, Phys. Rev. A 86, 041604(r) (2012).

[22] Bo Xiong, Jun-hui Zheng, and Daw-wei Wang, Phys. Rev. A 91, 063602 (2015).

[23] Biao Wu and Qian Niu, Phys. Rev. A 61, 023402 (2000).

[24] Fu-Quan Dou, Sheng-Chang Li, Hui Cao, Physics Letters A 376, 51 (2011).

[25] Y. Li, L. P. Pitaevskii, and S. Stringari, Phys. Rev. Lett. 108, 225301 (2012).

[26] Y. Li, G. I. Martone, L. P. Pitaevskii, and S. Stringari, Phys. Rev. Lett. 110, 235302 (2013).

[27] J. Y. Zhang, S. C. Ji, Z. Chen, L. Zhang, Z. D. Du, B. Yan, G. S. Pan, B. Zhao, Y. J. Deng, H. Zhai, S. Chen, and J. W. Pan, Phys. Rev. Lett. 109, 115301 (2012). 